\title{
IMPACTOS NEGATIVOS DA ENDOMETRIOSE NA QUALIDADE DE VIDA DA MULHER ACOMETIDA: UMA REVISÃO INTEGRATIVA DE LITERATURA
}

\begin{abstract}
Hanna Bezerra de Morais ${ }^{\text {; }}$ 0000-0002-6649-7289; Lourdes Manoela Lima Lisboa De Sousa ${ }^{2}$;0000-0002-55307814; Iara Alessandra Miranda Santos3; 0000-0003-4155-1768; Vitor Expedito Alves Ribeiro4; 0000-0002-92750954; Letícia Martins Barbosa Carvalho5; 0000-0001-8387-2688
\end{abstract}

\section{FILIAÇÃO}

(1) Universidade Estadual Do Maranhão (UEMA) - Centro de Estudos Superiores De Caxias, Medical Student

(2) Universidade Estadual Do Maranhão (UEMA) - Centro de Estudos Superiores De Caxias, Medical Student

(3) Universidade Estadual Do Maranhão (UEMA) - Centro de Estudos Superiores De Caxias, Medical Student

(4) Universidade Estadual Do Maranhão (UEMA) - Centro de Estudos Superiores De Caxias, Medical Student

(5) Universidade Estadual Do Maranhão (UEMA) - Centro de Estudos Superiores De Caxias, Medical Student

\section{AUTOR CORRESPONDENTE}

Hanna Bezerra de Morais; moraisbhanna@gmail.com Tv. São Benedito, $n^{\circ} 628$ - Caxias - MA. Universidade Estadual Do Maranhão (UEMA) - Centro de Estudos Superiores De Caxias; Acadêmica do $3^{\circ}$ período de Medicina

\section{MENSAGENS-CHAVE}

Sabe-se que, na endometriose, manifestações sintomatológicas diversas são comuns, dentre elas, dores muitas vezes incapacitantes e que interferem no cotidiano da mulher.

Tal condição pode afetar a qualidade de vida de sua portadora e ainda não há estratégias totalmente eficazes em seu tratamento.

Entender os impactos negativos da endometriose para a paciente é importante na compreensão da doença como um todo e na definição do tratamento adequado.

Estratégias terapêuticas mais efetivas, holísticas e acessíveis e seu diferencial ainda são pouco desenvolvidas, bem como os estudos em torno delas.

Urge que sejam desenvolvidos mais estudos sobre esse tema, a fim de obter-se abordagens que tornem a convivência com a doença mais tolerável à mulher.

\section{RESUMO}

INTRODUÇÃO: A endometriose é uma doença ginecológica benigna e crônica, caracterizada pela presença de tecido endometrial no exterior da cavidade uterina. O presente estudo busca entender como as possíveis manifestações sintomatológicas da endometriose podem afetar a qualidade de vida da mulher acometida e como o convívio com a doença pode se tornar mais tolerável à paciente. METODOLOGIA: Trata-se de uma revisão integrativa da literatura, realizada por meio de pesquisa nas bases PubMed, BVS e SCIELO, combinando os termos "Endometriose", "Qualidade de vida", "Vida Sexual", "Infertilidade", "Depressão", "Experiência feminina", "Sexualidade" e "Convivência". RESULTADOS: Os dados foram extraídos de 12 artigos com relação direta com a temática do estudo, selecionados nas bases utilizadas após a aplicação dos critérios de inclusão e exclusão. Os estudos mostraram que os sintomas da endometriose, como a dispareunia, a dor pélvica e a infertilidade, comprometem a realização de atividades cotidianas e que há diversos entraves que atrasam seu diagnóstico e tratamento. DISCUSSÃO: A análise dos resultados revela conformidade entre os artigos selecionados ao afirmarem que a sintomatologia da endometriose, ao interferir no cotidiano da portadora, pode impactar de forma negativa em sua qualidade de vida, bem como os entraves no diagnóstico e tratamento dessa patologia. CONCLUSÃO: É evidente que a convivência com a endometriose pode afetar negativamente diversos aspectos imprescindíveis na qualidade de vida da mulher acometida. 
Assim, torna-se necessário um tratamento multiprofissional holístico e individualizado para a mulher, de modo a prestar apoio humano e psicológico, além de incluir o parceiro e a família no tratamento e de envolver a paciente nas decisões terapêuticas. Ademais, deve-se investir em estudos sobre terapêuticas com maior eficácia para a maioria das mulheres portadoras de endometriose.

PALAVRAS-CHAVE: Endometriose; Impactos na Saúde; Qualidade de vida.

\begin{abstract}
INTRODUCTION: Endometriosis is a benign and chronic gynecological disease characterized by the presence of endometrial tissue outside the uterine cavity. The present study seeks to understand how the possible symptomatological manifestations of endometriosis may affect the quality of life of the affected woman and how living with the disease may become more tolerable to the patient. METHODOLOGY: This is an integrative review of the literature, carried out through research in pubmed, VHL and SCIELO databases, combining the terms "Endometriosis", "Quality of life", "Sexual Life", "Infertility", "Depression", "Female Experience", "Sexuality" and "Coexistence". RESULTS: The data were extracted from 12 articles with direct relation to the study theme, selected in the databases used after the application of the inclusion and exclusion criteria. Studies show that symptoms of endometriosis, such as dyspareunia, pelvic pain and infertility, compromise daily activities and that there are several obstacles that delay its diagnosis and treatment. DISCUSSION: The analysis of the results reveals conformity among the selected articles by stating that the symptomatology of endometriosis, by interfering in the daily life of the carrier, can negatively impact on their quality of life, as well as the obstacles in the diagnosis and treatment of this pathology. CONCLUSION: It is evident that living with endometriosis can negatively affect several essential aspects in the quality of life of the affected woman. Thus, it is necessary a holistic and individualized multiprofessional treatment for the woman, in order to provide human and psychological support, in addition to including the partner and the family in the treatment and involving the patient in therapeutic decisions. Furthermore, moreover, research should be invested in studies on therapies with greater efficacy for most women with endometriosis.
\end{abstract}

KEYWORDS: Endometriosis; Impacts in Health; Quality of Life.

\section{INTRODUÇÃO}

A endometriose é uma complexa doença ginecológica benigna e crônica, definida pela presença de tecido semelhante ao endométrio fora da cavidade uterina, acompanhada de inflamação crônica induzida por esses implantes ectópicos, que acometem toda a cavidade pélvica, incluindo peritônio, ovário, bexiga e/ou intestinos. Afeta de 10 a $15 \%$ das mulheres em idade reprodutiva e tende a ser diagnosticada em mulheres com crises de dor pélvica ou que tentam engravidar sem sucesso ${ }^{1}$.

Até o presente momento não existe uma hipótese específica para justificar o crescimento ectópico do tecido endometrial. Inúmeras investigações acerca dessa desordem ginecológica têm relacionado a sua ocorrência com distúrbios genéticos e imunológicos ${ }^{2}$. Apesar de ser considerada metastática benigna, exibe algumas características de doença maligna como, por exemplo, crescimento agressivo e invasão localizada ${ }^{3}$.

De acordo com Bulun ${ }^{4}$, apenas uma pequena parcela das pacientes com endometriose é assintomática (cerca de 3 a $22 \%$ ) e uma grande maioria apresenta sintomas variados, sendo que a dismenorreia, a dispareunia, a dor pélvica crônica, a irregularidade menstrual e a infertilidade são comumente evidenciadas ${ }^{4}$.

A endometriose apresenta sintomatologia variada e inespecífica. Isso ocorre na medida em que a manifestação sintomática da doença, quando presente, pode ser confundida com outros quadros clínicos e apresentar diferentes formas e intensidades em cada paciente, o que dificulta o diagnóstico ${ }^{5}$. Além disso, o reconhecimento da doença pode ser atrasado por fatores mais técnicos, como a dificuldade de acesso à laparoscopia,
Procedimento considerado hoje o mais eficiente no diagnóstico da endometriose ${ }^{6}$.

Tal retardo no diagnóstico da patologia é um empecilho para o estabelecimento de uma abordagem e tratamento adequados. Assim, prolonga a convivência da paciente com o sofrimento provocado pelos sintomas que, de acordo com Matta e Muller ${ }^{1}$, comprometem o cotidiano, causam impactos emocionais e atravessam diversas esferas da vida da mulher ${ }^{1}$. Ademais, o convívio com a dor pélvica crônica pode causar sérios prejuízos físicos, psíquicos e sociais, uma vez que, segundo Lorençatto $^{7}$, ela restringe e modifica o convívio diário da paciente com suas rotinas até então estabelecidas ${ }^{7}$.

Ao considerar o conceito delineado pela Organização Mundial da Saúde $(\mathrm{OMS})^{2}$, que estabelece qualidade de vida como "a percepção do indivíduo de sua posição na vida, no contexto da cultura e dos sistemas de valores nos quais ele vive e em relação a seus objetivos, suas expectativas, seus padrões e suas preocupações", é evidente que a endometriose possui impactos negativos sobre a qualidade de vida da mulher acometida ${ }^{2}$. Portanto, faz-se necessário buscar compreender esses impactos a fim de estabelecer estratégias de tratamento, protocolos e programas de saúde.

Frente a isso, o presente estudo pretende identificar problemáticas recorrentes no cotidiano da portadora de endometriose, compreender seus impactos na qualidade e em diversos âmbitos da vida da mulher acometida e analisar estratégias capazes de tornar o convívio com a condição mais tolerável à paciente.

\section{METODOLOGIA \\ 2.1. TIPO DE ESTUDO}

O estudo em questão consiste em uma revisão integrativa da 
literatura, através da qual realizou-se síntese, análise, comparação e interpretação de conhecimentos científicos anteriormente produzidos sobre o tema "IMPACTOS DA ENDOMETRIOSE NA QUALIDADE DE VIDA DA MULHER ACOMETIDA".

A realização da revisão de literatura do tipo integrativa no presente estudo permite reunir, sintetizar e analisar de forma crítica conhecimentos previamente produzidos e publicados sobre os impactos da endometriose na qualidade de vida da mulher acometida.

\subsection{IDENTIFICAÇÃO DO TEMA E SELEÇÃO DA QUESTÃO DE PESQUISA}

A temática sobre o efeito da endometriose na qualidade de vida das mulheres acometidas determinou a construção da estratégia PICO, conforme instruído por Haynes, que representa um acrônimo para Paciente/população $(P)$, Interesse (I), Contexto (Co) ou Comparação (C) e Outcome (O), ${ }^{25}$ a qual foi utilizada para a localização de estudos relevantes que respondessem à seguinte pergunta dessa revisão integrativa da literatura: De que forma a endometriose e os entraves acerca do seu diagnóstico e prognóstico impactam nos diversos aspectos da função sexual feminina, da sua capacidade reprodutiva, da sua saúde mental e da sua produtividade no trabalho, entre outros tantos fatores físicos e sociais que são imprescindíveis para a qualidade de vida das mulheres acometidas?

Assim, realizou-se uma busca, no período de março a junho de 2020, a partir do descritor "Endometriose" associado aos descritores "Infertilidade", "Disfunção sexual", "Relações sociais", "Saúde mental", "Impactos", "Qualidade de vida", "Diagnóstico" e "Tratamento" nos idiomas português e inglês, obtidos através dos descritores em Ciências da Saúde (DeCS) e em Medical Subject Headings (Mesh). A pesquisa foi realizada por três pesquisadores, sem 0 conhecimento dos artigos selecionados pelos outros, de modo a realizar uma seleção de artigos que, em consenso, foram considerados relevantes.

Os descritores e palavras-chave foram usados em PudMed da National Library of Medicine, SCIELO (Scientific Electronic Library Online) e BVS (Biblioteca Virtual em Saúde), bases de dados amplamente utilizadas na área da saúde. Durante a pesquisa, os termos utilizados foram associados e classificados nos bancos de dados, a fim de encontrar uma estratégia de busca eficaz.

\subsection{ESTABELECIMENTO DOS CRITÉRIOS DE INCLUSÃO E EXCLUSÃO}

Após as buscas nas bases de dados, com a utilização das associações Endometriosis AND Quality of life; Endometriosis AND Sexual life; Endometriosis AND Infertility; Endometriosis AND Depression; Endometriosis AND Feminine experience e Endometriose e qualidade de vida; Endometriose e sexualidade; Endometriose e infertilidade; Endometriose e depressão; Endometriose e convivência, foram encontrados 862 artigos. Mediante aplicação dos critérios de inclusão (publicados entre janeiro de 2000 e maio de 2020; têm como tema principal a endometriose; classificados como revisão sistemática, casocontrole retrospectivo, caso-controle prospectivo, estudos de coorte, ensaios clínicos controlados randomizados ou quase randomizados; de acesso livre e gratuito) e dos critérios de exclusão (artigos com texto completo indisponível, artigos que não foram escritos em inglês ou em português, artigos do tipo relato de caso e editoriais), restaram 135 publicações.

Dessas, 12 publicações apresentavam metodologia, temática e objetivos condizentes com o objetivo dessa pesquisa de analisar o impacto geral da endometriose na vida da mulher acometida. O processo de seleção dos estudos foi especificado em um fluxograma (Anexo 1).

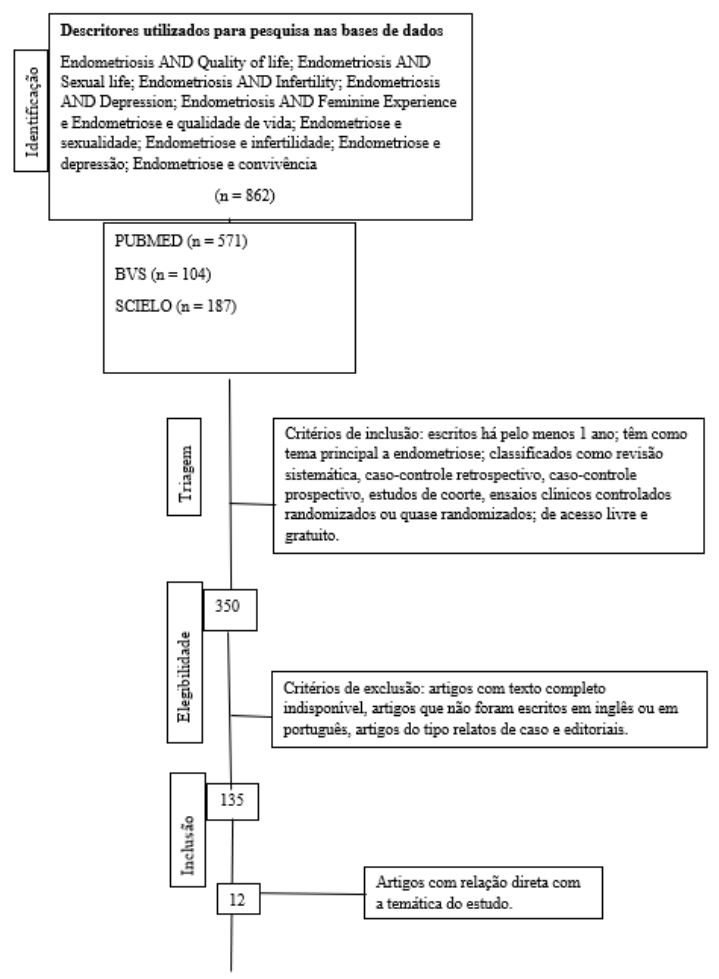

Anexo 1: Fluxograma do processo de seleção de estudos para construção da revisão

Fonte: Pesquisa direta, 2021

\subsection{PROCESSO DE EXTRAÇÃO DE DADOS}

Após a leitura minuciosa de cada um dos artigos selecionados, foi iniciada a extração de dados relevantes para a construção desta revisão. Para esta tarefa, foram usados formulários eletrônicos no software Microsoft Excel para compilação de dados. Desse processo participaram 4 autores que fizeram uma seleção de dados de forma independente, com o objetivo de garantir unanimidade em relação à escolha das informações. Também foi estabelecido um método em casos de discordâncias: essas devem ser analisadas pelo quinto pesquisador. Importante ressaltar que foi realizado 0 mascaramento dos investigadores para autores, instituições e revistas dos estudos escolhidos, a fim de que os avaliadores não fossem influenciados por conceitos prévios a respeito dos artigos.

\section{RESULTADOS}

Foram selecionados 12 artigos considerados relevantes para a 
Tabela 1. Resultados da pesquisa bibliográfica

\begin{tabular}{cccccc}
\hline ESTUDO & ANO & PAISS & $\begin{array}{c}\text { BASE } \\
\text { DE } \\
\text { DADOS }\end{array}$ & METODOLOGIA & OBJETIVO DO \\
ESTUDO & PRINCIPAIS RESULTADOS
\end{tabular}

\begin{tabular}{|c|c|c|c|c|c|}
\hline $\begin{array}{c}\text { Ramos et } \\
\text { al. }{ }^{12}\end{array}$ & 2018 & Brasil & SCIELO & $\begin{array}{c}\text { Estudo } \\
\text { exploratório quali } \\
\text { tativo }\end{array}$ & $\begin{array}{c}\text { Identificar o } \\
\text { conhecimento de } \\
\text { mulheres que } \\
\text { convivem com } \\
\text { endometriose a } \\
\text { respeito da } \\
\text { doença e ponderar } \\
\text { suas percepções } \\
\text { sobre a qualidade } \\
\text { de vida pós- } \\
\text { diagnóstico. }\end{array}$ \\
\hline $\begin{array}{l}\text { Matta \& } \\
\text { Muller }^{1}\end{array}$ & 2006 & Brasil & SCIELO & $\begin{array}{c}\text { Análise textual } \\
\text { qualitativa }\end{array}$ & $\begin{array}{c}\text { Conhecer as } \\
\text { vivências de } \\
\text { mulheres com } \\
\text { diagnóstico de } \\
\text { endometriose } \\
\text { associadas à } \\
\text { doença. }\end{array}$ \\
\hline
\end{tabular}

As participantes (30-39 anos) da pesquisa não conhecem a doença que lhes aflige, vivem em companhia da dor e da possível infertilidade. A maioria já sofreu abortamento.

Foram identificadas categorias que descrevem a magnitude da influência da endometriose sobre a vida cotidiana das mulheres, como seus desdobramentos afetivos, familiares, profissionais e de relação com a classe médica. Também, buscou-se identificar aquilo que estas mulheres entendem como sentido e significado da endometriose em suas vidas.

\begin{tabular}{|c|c|c|c|c|}
\hline $\begin{array}{c}\text { Oliveira; } \\
\text { Brilhante; Lo } \\
\text { urinho }{ }^{11}\end{array}$ & 2018 Brasil & BVS & $\begin{array}{c}\text { Estudo } \\
\text { transversal }\end{array}$ & $\begin{array}{c}\text { Compreender a } \\
\text { relação existente } \\
\text { entre a ocorrência } \\
\text { de endometriose e } \\
\text { o sofrimento } \\
\text { psíquico presente } \\
\text { nas mulheres. }\end{array}$ \\
\hline
\end{tabular}

Estudo
observacional
transversal
transversal

$\begin{gathered}\text { Florentino et } \\ \text { al. }{ }^{21}\end{gathered}$

Avaliar a
existência de
associação entre
os achados ultrassonográficos e os fatores epidemiológicos e clínicos com os resultados obtidos no questionário EHP-30 em mulheres com endometriose ovariana.

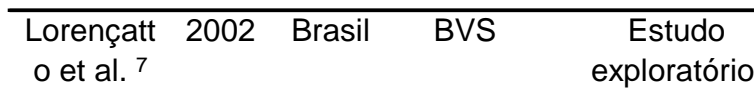

Avaliar a frequência de depressão em pacientes com endometriose e queixa atual de dor pélvica para determinar a necessidade ou não de atendimento psicológico.
A demora no diagnóstico e a

busca pelo tratamento das dores incapacitantes são condições que contribuem para a alteração do humor da mulher, levando a uma reação estressante. Mesmo após esse processo, a mudança de humor e a ansiedade são frequentes. É necessária uma abordagem holística para lidar com a doença.

Infertilidade estava presente em $48,4 \%$ das pacientes estudadas. Do total de casos, $80,6 \%$ eram sintomáticas e queixaram-se principalmente de dor acíclica, $79 \%$ de dismenorreia e $61,3 \%$ de dispareunia, refletindo a influência negativa da endometriose sobre a qualidade de vida das pacientes.
Identificou-se a presença de depressão em $92 \%$ das pacientes avaliadas, sendo $56 \%$ de intensidade moderada a grave. pacientes avaliadas, sendo $56 \%$ de intensidade moderada a grave. Apesar de $66 \%$ das pacientes relatarem o uso de medicação, não foi Apesar de 66\% das pacientes relatarem o uso de medicação, não foi observada correlação significativa entre a presença de depressão com o uso de hormônios 


\begin{tabular}{|c|c|c|c|c|c|c|}
\hline ESTUDO & ANO & PAÍS & $\begin{array}{l}\text { BASE } \\
\text { DE } \\
\text { DADO } \\
\text { S }\end{array}$ & METODOLOGIA & $\begin{array}{l}\text { OBJETIVO DO } \\
\text { ESTUDO }\end{array}$ & PRINCIPAIS RESULTADOS \\
\hline $\begin{array}{c}\text { Bento \& } \\
\text { Moteira } \\
16\end{array}$ & 2018 & Brasil & BVS & $\begin{array}{c}\text { Estudo } \\
\text { exploratório }\end{array}$ & $\begin{array}{l}\text { Discutir os significados } \\
\text { atribuídos por mulheres } \\
\text { à dor causada pela } \\
\text { endometriose }\end{array}$ & $\begin{array}{l}\text { As mulheres com endometriose sofrem } \\
\text { banalização da dor crônica vivenciada. }\end{array}$ \\
\hline $\begin{array}{l}\text { Trovó- } \\
\text { Marqui et } \\
\text { al. }{ }^{22}\end{array}$ & 2015 & Brasil & BVS & $\begin{array}{l}\text { Revisão de } \\
\text { literatura }\end{array}$ & $\begin{array}{l}\text { Avaliar a função sexual } \\
\text { das pacientes com } \\
\text { endometriose, bem } \\
\text { como a participação de } \\
\text { fatores de risco. }\end{array}$ & $\begin{array}{l}\text { Os estudos mostraram que as pacientes } \\
\text { com endometriose exibem } \\
\text { comprometimento da função sexual e o } \\
\text { tratamento farmacológico e/ou cirúrgico foi } \\
\text { eficaz na melhora dessa variável. }\end{array}$ \\
\hline $\begin{array}{l}\text { Dias } \\
\text { Vila et } \\
\text { al. }{ }^{10}\end{array}$ & 2010 & Brasil & $\begin{array}{c}\text { SCIEL } \\
\text { O }\end{array}$ & $\begin{array}{c}\text { Estudo } \\
\text { exploratório }\end{array}$ & $\begin{array}{l}\text { Discutir aspectos do } \\
\text { cotidiano da paciente } \\
\text { com endometriose, } \\
\text { considerando o } \\
\text { sentimento feminino } \\
\text { perante o diagnóstico e } \\
\text { o tratamento. }\end{array}$ & $\begin{array}{l}\text { Insegurança e ansiedade foram os } \\
\text { sentimentos mais frequentes frente ao } \\
\text { diagnóstico, mas, uma vez iniciado o } \\
\text { tratamento, a maioria das participantes } \\
\text { relatou bem-estar como sentimento } \\
\text { predominante. }\end{array}$ \\
\hline
\end{tabular}

\begin{tabular}{ccccc}
\hline $\begin{array}{c}\text { Arruda et } \\
\text { al. }\end{array}$ & 2008 & Brasil & Pubmed & $\begin{array}{c}\text { Estudo de coorte } \\
\text { retrospectivo }\end{array}$
\end{tabular}

Avaliar o tempo entre 0 início dos sintomas e o diagnóstico de endometriose, e identificar os fatores associados ao retardo diagnóstico em um grupo de mulheres brasileiras.

O tempo médio decorrido desde o início dos sintomas até o diagnóstico foi de 7,0 anos. Quanto mais jovens as mulheres no início dos sintomas, maior o período para o diagnóstico: o atraso médio foi de 12,1 anos em mulheres com 19 anos ou mais, e 3,3 anos em mulheres com idade 30 anos ou mais. O tempo médio entre o início dos sintomas e o diagnóstico foi de 4,0 anos para mulheres com queixa de infertilidade, mas 7,4 anos para aquelas com dor pélvica.

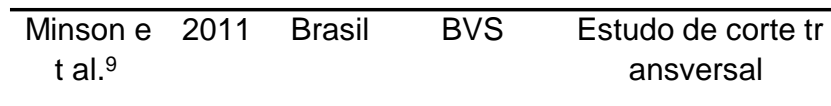

Examinar a relação entre aspectos clínicos e qualidade de vida em um grupo de pacientes com endometriose

$63 \%$ das pacientes estavam no estágio 3 ou 4 de endometriose, $36 \%$ das pacientes sofriam de dismenorreia severa ou incapacitante e a intensidade média da dor, segundo escala visual numérica, foi de 5,6 (DP=3,5)

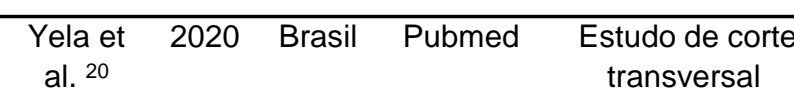

Descrever características clínicas e sociodemográficas de mulheres com endometriose profunda infiltrativa e avaliar sua qualidade de vida dentro de 6 meses de tratamento clínico.

\begin{tabular}{ccccc}
\hline $\begin{array}{c}\text { Nnoaham } \\
\text { et al. } 19\end{array}$ & Estad & Pubmed & Estudo \\
& os & & transversal \\
& & Unido & & \\
& &
\end{tabular}

Avaliar o impacto da endometriose na qualidade de vida relacionada à saúde e na produtividade no trabalho.
$50 \%$ das participantes apresentaram dismenorreia, 57\% dispareunia e 50\% dor pélvica crônica. O SF-36 e o EHP30 mostraram comprometimento da qualidade de vida destas mulheres. No SF-36, os piores domínios foram os aspectos emocionais e a autoestima e disposição, enquanto que no EHP-30 foram o bem-estar social, a infertilidade e as relações sexuais.

Houve atraso entre o início dos sintomas e o diagnóstico de endometriose, que foi mais longo em centros financiados pelo estado. O retardo foi associado ao número de sintomas pélvicos e a um IMC mais alto. A QVRS foi reduzida em mulheres afetadas em comparação àquelas com sintomas semelhantes $\mathrm{e}$ sem endometriose. Cada mulher afetada perdeu em média 10,8 horas de trabalho semanais, principalmente devido à redução da eficácia durante o trabalho. 
construção do presente artigo, uma vez que apresentam resultados e conclusões com dados importantes a serem considerados na avaliação do acometimento da endometriose na qualidade de vida da mulher. Entre os 12 estudos, três estavam na base de dados SCIELO, quatro na base de dados PubMed e 5 na BVS. Além disso, 11 dos artigos selecionados estão na língua portuguesa e apenas um dos artigos é de língua inglesa.

Entre os principais resultados encontrados nos estudos escolhidos, tem-se que Matta e Muller ${ }^{1}$, após entrevistarem quatro mulheres diagnosticadas com endometriose e transcreverem as entrevistas, realizaram uma análise textual qualitativa. Com isso, diversos pontos de destaque nos relatos das portadoras foram identificados. Dentre eles, pode-se citar 0 fato de que a convivência com a endometriose anterior ao diagnóstico é um desafio para a mulher, tanto pela dor cíclica ou aguda de origem até então desconhecida quanto pela dificuldade de comunicação que existe entre médico e paciente.

Nesse estudo, outro ponto relevante é a demora no diagnóstico da endometriose, relatada por 3 das 4 mulheres entrevistadas, uma vez que essa doença em fase aguda costuma ser confundida com outros quadros clínicos, como a apendicite. Menciona-se, ainda, como impasse para o diagnóstico e o tratamento adequado da endometriose, a dificuldade em encontrar um médico que não banalize ou desacredite as dores relatadas pela paciente.

Já Arruda et al. ${ }^{8}$ realizaram um estudo no qual foram entrevistadas 200 mulheres brasileiras com endometriose confirmada cirurgicamente. Descobriu-se que o tempo médio decorrido entre o início dos sintomas e o diagnóstico da endometriose era de 7 anos. $O$ estudo ${ }^{8}$ revelou, também, que a idade é inversamente proporcional ao tempo para buscar ajuda profissional e para a obtenção do diagnóstico. Cita-se, também, a dificuldade de acesso à laparoscopia como método de diagnóstico.

O estudo de Paulo \& Martha ${ }^{16}$ foi realizado com 20 mulheres, de 21-48 anos, diagnosticadas com endometriose a partir de entrevistas. Esse demonstrou um grande atraso no diagnóstico das participantes, que variaram de dois anos a 26 anos, com média de 12 anos, concluindo que se trata de uma doença com significativo atraso no diagnóstico ${ }^{16}$.

No estudo de Lorençatto et al. $^{7}$, foram avaliadas 50 pacientes, entre 24 e 48 anos, com diagnóstico confirmado de endometriose e presença de dor pélvica crônica há pelo menos seis meses, através de uma entrevista individual feita por uma psicóloga. Foi utilizado o Inventário de Depressão de Beck ("Beck Depression Inventory"; BDI) para investigar a prevalência da depressão. A partir dessas entrevistas, foi possível identificar a presença de depressão em $92 \%$ das pacientes avaliadas.

Enquanto que Oliveira, Brilhante e Lourinho11 realizaram entrevistas narrativas com 11 mulheres portadoras de endometriose em uma maternidade em Fortaleza, no Ceará, de setembro de 2016 a janeiro de 2017. Após essa coleta de dados, analisou-se a relação da endometriose com o sofrimento psíquico das pacientes. Observou-se, então, que a endometriose compromete a qualidade de vida da mulher ao provocar não apenas sofrimento físico, como também psicológico.

Já o estudo de Ramos et al. ${ }^{12}$ procurou avaliar o conhecimento das participantes sobre a doença que Ihes afligia, constatando, assim, que a maioria não conseguia elaborar um conceito que se aproximasse do real significado da endometriose, relatando apenas a presença de dores fortes e frequentes. Segundo as entrevistadas, muitos dos médicos frequentados por elas não demonstraram preocupação em informá-las adequadamente sobre seu diagnóstico, tratamento e prognóstico, fazendo com que a maioria buscasse esclarecer suas dúvidas na internet. No entanto, grande parte das mulheres expressou a sensação de alívio após o diagnóstico de endometriose.

Dias Vilas et al. ${ }^{10}$ convidaram 40 mulheres com diagnóstico de endometriose, com idade entre 24 a 44 anos, a responder um questionário. Entre elas, a queixa mais frequente foi a ocorrência de cólicas, principal razão que motivou o aconselhamento médico. Quando perguntadas sobre as mudanças após o início do tratamento, 17 participantes referiram ter sofrido mudanças negativas em sua qualidade de vida, como: ganho excessivo de peso, efeitos colaterais de medicações e estresse ligado à antecipação da maternidade. $\mathrm{E}$ quatro mulheres interromperam 0 tratamento médico, alegando frustração com ele.

Trovó-Marqui et al.22 realizou uma pesquisa bibliográfica sobre a relação entre disfunção sexual e endometriose. Em seu estudo, a maioria dos artigos analisados avaliaram o efeito do tratamento da endometriose na disfunção sexual, sendo o tratamento cirúrgico o mais frequente avaliado. Os questionários SAQ, FSFI e GSSI foram os mais utilizados, sendo empregados em $60 \%$ dos estudos.

Já o estudo realizado por Minson et al.9 contou com a participação de 130 mulheres avaliadas por meio do questionário de qualidade vida SF-36, os resultados sugerem que a saúde geral das pessoas com endometriose não é apenas determinada pelo estadiamento da doença, tempo de manifestação dos sintomas e intensidade da dor, mas também por outros aspectos, como fatores psicossociais.

Em estudo realizado por Nnoaham et al.19, foram recrutadas, em 16 hospitais de 10 países (Argentina, Brasil, Estados Unidos, Irlanda, Inglaterra, Bélgica, Itália, Espanha, Nigéria e China), mulheres de 18 a 45 anos com laparoscopia marcada para investigar dor pélvica associada à endometriose ou para serem esterilizadas. Na semana anterior à cirurgia, as participantes responderam um questionário de 67 itens sobre as queixas que apresentavam e seus impactos na Qualidade de Vida Relacionada à Saúde (QVRS), atividades diárias, histórico médico e recursos de atendimento à saúde. Foram aplicados o questionário Short Form-36 version 2 (SF36v2), usado para medir a QVRS; e o questionário Work Productivity and Activity Impariment (WPAI:GH), a fim de avaliar a frequência de mulheres portadoras de endometriose sintomáticas e contratadas no trabalho e os impactos dos 
sintomas na realização de atividades. O impacto dos sintomas sobre a produtividade no trabalho e demais atividades foram mensuradas em uma escala de 0 a 10 . Ademais, três grupos foram observados: mulheres com endometriose; mulheres de controle sintomático sem endometriose e mulheres de controle de esterilização sem endometriose $^{19}$.

Observou-se um atraso médio de 6,7 anos entre o início dos sintomas e o diagnóstico cirúrgico da endometriose. Esse atraso se mostrou associado com a quantidade de sintomas pélvicos e a um maior IMC. Mulheres sintomáticas com endometriose apresentaram Qualidade de Vida Relacionada à Saúde reduzida quando comparadas a mulheres que apresentavam os mesmos sintomas, mas sem endometriose. Além disso, cada mulher acometida mostrou uma perda média de 10,8 horas de trabalho semanais, devido à queda da produtividade causada pela doença ${ }^{19}$.

Yela et al. ${ }^{20}$ realizou entrevistas com 58 mulheres, com 2649 anos, diagnosticadas com endometriose profunda e que deram início ao tratamento. Para avaliar a qualidade de vida, foram utilizados dois questionários: o Endometriosis Health Profile Questionnaire (EHP-30), um questionário mais específico, que aborda alguns pontos como a função sexual; a questão da maternidade; a relação entre médico e paciente; e o Short Form - 36 (SF-36), que é mais genérico. De acordo com o EHP-30, tem-se um maior comprometimento na qualidade de vida nos domínios sociais, como bem-estar social, suporte social, capacidade de enfrentamento da doença e fertilidade.

O estudo de Florentino et al.21, 62 mulheres brasileiras, com faixa etária de 20 a 54 anos, sexualmente ativas em idade reprodutiva e com diagnóstico de endometriose foram entrevistas através do questionário EHP-30, no qual a menor pontuação indica melhor qualidade de vida. Destas $48,4 \%$ dos pacientes estudados eram inférteis.

Os resultados mais relevantes, juntamente com os objetivos principais, metodologia e outras informações importantes (base de dados onde foi encontrada, país e ano de publicação) sobre cada um dos estudos selecionados foi sintetizada na Tabela 1.

\section{DISCUSSÃO}

A partir da análise e interpretação dos resultados, pode-se concluir que a endometriose impacta negativamente em diversos aspectos da qualidade de vida da mulher: sua vida social, profissional, sexual, sua saúde mental e suas relações interpessoais e visão sobre si mesma. Ademais, percebe-se a existência de empecilhos que atrasam diagnóstico e tratamento da doença, que colaboram para estender o sofrimento de sua portadora. Portanto, é necessário que sejam os obstáculos ao diagnóstico e tratamento da endometriose sejam superados, e que sejam traçadas estratégias terapêuticas holísticas e capazes de mitigar os efeitos negativos da endometriose sobre a qualidade de vida das pacientes acometidas.

\section{Dificuldade na obtenção de diagnóstico}

Devido ao atraso que frequentemente ocorre no diagnóstico da endometriose, a descoberta da doença não maligna como causadora dos sintomas representa uma forma de alívio para a portadora ${ }^{1}$. De início é comum que, devido à ausência de diagnóstico ou a uma associação entre o grau de severidade da doença e seus sintomas anatômicos, o profissional de saúde interprete a dor da paciente como psicogênica, bem como relatado por uma das entrevistadas no estudo de Matta e Muller ${ }^{1}$. Muitas mulheres foram apontadas como "loucas" por aqueles que com elas convivem devido à dificuldade encontrada pelos médicos em diagnosticar a doença ${ }^{12}$.

A relação de proporcionalidade inversa entre a idade da mulher e o tempo para obtenção de ajuda profissional e de um diagnóstico ${ }^{8}$ está relacionada a diversos fatores, a mencionar: pressuposto médico de que a dor menstrual é algo necessariamente fisiológico; o fato de adolescentes serem menos convincentes quando comparadas a mulheres mais velhas ao reportar seus sintomas; desconforto de pacientes mais jovens em relatar dor durante a relação sexual; maior dificuldade de realização de exame físico em adolescentes, em especial naquelas sexualmente inativas.

Ademais, a laparoscopia, método considerado o mais eficiente no diagnóstico da endometriose, é pouco acessível $^{8}$, fato que retarda a descoberta da doença. Por ser um procedimento oneroso em redes privadas, muitas mulheres recorrem aos serviços públicos de saúde para sua realização, o que resulta em longas listas de espera. Além disso, a laparoscopia ainda não está disponível em todos os hospitais, em especial na rede pública (na qual, por vezes, ainda há uso da laparotomia), e os ginecologistas podem não possuir familiaridade com o procedimento e relutar em encaminhar suas pacientes para centros especializados.

\section{Impacto na saúde mental das pacientes:}

- Presença de sintomas depressivos

$\mathrm{Na}$ endometriose, observa-se que a depressão está relacionada não só com os sintomas provocados pela doença, mas também com as características emocionais e cognitivas próprias de cada paciente ${ }^{7}$. Sendo que, como foi destacado no mesmo estudo, a endometriose afeta a qualidade de vida da mulher, ocasionando um comprometimento das suas funções vitais e das suas percepções cognitivas, que facilmente pode levar a um quadro depressivo. Esse efeito interfere ainda no tratamento das pacientes, gerando assim, resultados menos satisfatórios no controle dos sintomas, podendo ainda intensificar o quadro de depressão, o que se apresenta como um ciclo vicioso.

Também, a negligência e banalização da dor pelos profissionais da saúde gera sofrimento na paciente ${ }^{1,11}$. Pontua-se que o estado emocional da mulher pode intensificar ou enfraquecer a doença e seus impactos sobre a vida profissional, social e sexual da portadora ${ }^{11}$ e que é frequente a ocorrência de depressão em pacientes com endometriose $\mathrm{e}^{7,11}$.

\section{- Sofrimento psíquico com o diagnóstico}

No estudo de Dias Vilas et al.10, emoções negativas foram relacionadas à percepção das mulheres da infertilidade e do sentimento irracional de culpa por não terem prevenido a doença, atendendo-se por uma necessidade de maior 
acompanhamento psicológico à mulher infértil. $\mathrm{O}$ sentimento positivo mais relatado foi alívio (8 participantes) em tomar conhecimento de um diagnóstico que explicava sua condição, o que confirma o resultado encontrado por Ramos et al. ${ }^{12}$ e por Matta e Muller ${ }^{1}$.

\section{- Necessidade de apoio emocional durante diagnóstico e tratamento}

Frequentemente, constatou-se carência de apoio por parte do parceiro ${ }^{10}$, o que confirma a necessidade de compreensão evidenciada também em Ramos et al. ${ }^{12} \mathrm{O}$ apoio do parceiro, da família e dos amigos é importante e sua ausência pode favorecer transtornos emocionais, segundo Cunha et $\mathrm{al}^{13}$.

\section{Impacto da infertilidade no psicológico das pacientes}

No estudo de Dias Vilas et al. ${ }^{10}$, todas as mulheres estavam em tratamento por não conseguirem engravidar, o que confirma que a maternidade continua um aspecto importante da realização da identidade feminina ${ }^{15}$, e a presença de obstáculos para alcançá-la pode provocar sentimentos conflitantes em relação a si mesma1.

\section{- Impacto psicológico da dor}

Para as portadoras de endometriose, a dor apresenta papel principal, sendo descrita com grande intensidade, sofrimento e capacidade de levar ao afastamento do convívio social, profissional e familiar e, até mesmo, do impulso de viver ${ }^{10,11}$. A maioria relatou que a dor rouba-lhes a autonomia. De acordo com Helman ${ }^{17}$, dor é parte inseparável do cotidiano. Assim, essas mulheres relataram a necessidade de aprenderem a conviver com a dor.

\section{Repercussão da endometriose na atividade sexual}

Na pesquisa de Trovó-Marqui et al22, o questionário FSFI foi empregado em 4 estudos e avalia seis domínios da função sexual: desejo, excitação, lubrificação, orgasmo, satisfação e dor. Em um desses estudos, realizado com grupo controle, não houve diferença significativa entre os escores das mulheres com endometriose e das que não tinham nenhuma complicação ginecológica ou outra comorbidade. Segundo as autoras desses estudos, uma possível justificativa é que as pacientes com endometriose, embora apresentassem dispareunia profunda, minimizam esse sintoma devido ao desejo de engravidarem ${ }^{24}$. Essa hipótese pode ser reforçada pelo estudo de Paulo \& Martha ${ }^{16}$, que observou a "necessidade de aceitar a dor", relatada pelas mulheres.

De acordo com um dos estudos $23,69 \%$ das pacientes evitavam o sexo devido à dor e $19 \%$ não estavam ativas por causa da dor. A prevalência de disfunção sexual em endometriose variou entre os estudos observados de $32 \%$ a $73 \%$. Um estudo 22 mostrou que apenas nos casos mais avançados de endometriose tinha-se comprometimento da sexualidade, o que pode explicar essa variação na prevalência.

\section{- Impacto da endometriose na qualidade de vida}

Quanto ao impacto da endometriose na qualidade de vida dessas mulheres, segundo os dados coletados nesse estudo, a maior dificuldade encontrada foi conviver com a dor. Vários estudos, como Donatti et al.14, já revelaram a influência da intensidade da dor causada pela endometriose com a depressão e com o estresse. A maioria das mulheres demonstrou também o intenso desejo em procriar e as dificuldades enfrentadas nesse processo, constatando que a presença infertilidade afeta diretamente na saúde dessas mulheres, principalmente quando o parceiro não apresenta compreensão.

Durante a entrevista de Ramos et al.12, também foi perguntado às participantes sobre as mudanças que a endometriose acarretou em suas vidas e suas expectativas quanto ao assunto e a maioria absoluta relatou que a endometriose só trouxe desvantagens. Muitas delas alegaram interferência em sua vida profissional, sexual, na convivência familiar e no seu psicológico, concluindo que a endometriose diminui a qualidade de suas vidas.

A partir do estudo de Minson et al ${ }^{9} \cdot$, tem-se que a saúde das mulheres com endometriose se relaciona não apenas com aspectos orgânicos da doença, como sua manifestação sintomática, sua extensão e o tempo de apresentação de sintomas, mas também por aspectos psicossociais. Essa observação vai ao encontro da associação entre endometriose e fatores psíquicos presente no estudo de Oliveira; Brilhante e Lourinho ${ }^{11}$.

Nesse estudo, os escores obtidos por as participantes ${ }^{9}$ são inferiores se comparados a populações sadias ${ }^{18}$. Porém, foram levemente superiores a outras pesquisas sobre a qualidade de vida das mulheres com endometriose. Esse resultado pode estar relacionado com 0 alto nível socioeconômico da população participante neste estudo, pois sua renda permite acesso a uma terapêutica mais eficiente. De forma geral, os escores dos pacientes nas escalas do SF-36 variaram entre o percentil 51 na dimensão vitalidade e 80 na dimensão capacidade funcional, sugerindo que a endometriose impacta de forma diferente em várias dimensões da vida da mulher ${ }^{9}$.

Os domínios psicológicos, como autoestima, disposição e aspectos emocionais, afetaram mais intensamente a qualidade de vida dessas mulheres do que os domínios físicos do SF-36. As divergências entre estudos que analisam a associação entre a depressão e a dor crônica se devem à forma individual de cada paciente de se adaptar à situação de dor, podendo desenvolver ou não uma depressão ${ }^{20}$.

Observou-se que o tratamento médico ajuda a mulher a melhorar em alguns itens, pois foi eficaz no controle da dor. Embora o manejo não seja eficaz na obtenção de uma excelente qualidade de vida, ainda exerce um impacto significativamente negativo na qualidade de vida ${ }^{20}$.

Ademais, é elevado o índice de infertilidade em mulheres com endometriose. No estudo de Florentino et al. ${ }^{21,} 48,4 \%$ dos pacientes estudados eram inférteis, estando de acordo com outros estudos realizados ${ }^{13,17}$.

\section{- Impacto do tratamento na qualidade de vida}

Quanto ao tratamento, Oliveira; Brilhante e Lourinho'11 o descrevem como uma nova fase na convivência da mulher com sua endometriose. Essa fase pode ter sua continuidade dificultada pelos seus efeitos negativos em potencial. O estudo ressalta que o tratamento deve abordar a endometriose a partir de um manejo integral, visando não apenas a patologia orgânica, como também apoio humano dos profissionais da saúde envolvidos, suporte psicológico e avaliação do estado emocional da paciente.

No estudo de Dias Vilas et al. ${ }^{10} 42,5 \%$ das entrevistadas relataram impactos negativos do tratamento em sua qualidade de vida, e quatro delas deixaram de seguir o 
tratamento por se sentirem frustradas. Dessa forma, notase, em concordância com o estudo de Oliveira; Brilhante e Lourinho ${ }^{11}$ a importância de um acompanhamento holístico e humano por parte do profissional de saúde, a fim de fornecer o apoio emocional necessário para que paciente persista no tratamento.

As principais limitações deste estudo consistem na restrição dos resultados causada pelos critérios de inclusão e exclusão e pelo uso de descritores determinados durante a busca nas bases de dados utilizadas. Além disso, não foram encontrados estudos aprofundados e com maiores dados sobre os diferenciais de abordagens terapêuticas mais abrangentes e holísticas no tratamento da endometriose; sobre a evolução psíquica da mulher no decorrer do tratamento; sobre o papel da relação médicopaciente ou sobre formas mais acessíveis e democráticas de tratamento.

\section{CONCLUSÃO}

Conforme os estudos analisados, é evidente que a convivência com a endometriose, suas decorrências e os entraves ao seu diagnóstico e tratamento, tanto anterior quanto posterior à descoberta da doença, interfere em diversos aspectos da vida social, sexual, emocional e profissional de sua portadora. Assim, tal condição pode impactar negativamente na qualidade de vida da mulher acometida.

É necessária uma conscientização dos profissionais da saúde, para que estejam atentos à possibilidade de endometriose quando na presença de sintomas característicos dessa doença. Além disso, é de suma importância que seja desenvolvida uma abordagem multiprofissional holística e individualizada para a mulher, de modo a prestar apoio humano e psicológico, levar em conta as particularidades de cada caso, incluir o parceiro e a família no tratamento e envolver a paciente nas decisões terapêuticas.

Por fim, é essencial que sejam desenvolvidos novos estudos a fim de aperfeiçoar as técnicas de tratamento existentes para a endometriose, uma vez que, apesar da grande eficácia da intervenção cirúrgica, abordagens como a hormonal não se mostraram igualmente efetivas. Ainda, por vezes, o tratamento impacta negativamente na qualidade de vida da mulher, o que gera frustração e dificulta a adesão da paciente. Portanto, deve-se buscar estratégias terapêuticas de maior efetividade no combate aos sintomas da endometriose e que permitam à portadora da doença uma convivência mais tolerável com sua condição.

\section{CONFLITO DE INTERESSE}

Os autores declaram que não há nenhum conflito de interesse presente no estudo.

\section{FINANCIAMENTO}

Os autores declaram que não houve fontes de financiamento.

\section{REFERÊNCIAS}

1. MATTA AZ,MULLER MC. Uma análise qualitativa da convivência da mulher com sua endometriose. Psicologia, Saúde e Doenças [Internet]. 2006;7(1):57$72 . \quad$ Recuperado

https://www.redalyc.org/articulo.oa?id=36270104

de:

2. Riccio LDGC, Santulli $P$, Marcellin L, Abrão MS, Batteux F, Chapron C. Immunology of endometriosis. Best Pract Res Clin Obstet Gynaecol. [Internet] 2018 Jul;50:39-49. Disponível em: https://doi.org/10.1016/j.bpobgyn.2018.01.010

3. Kyama CM, Mihalyi A, Gevaert O, Waelkens E, Simsa P, Van de Plas R, Meuleman C, De Moor B, D'Hooghe TM. Evaluation of endometrial biomarkers for semiinvasive diagnosis of endometriosis. Fertil Steril [Internet]. 2011 Mar 15;95(4):1338-43. Disponível em: https://doi:10.1016/j.fertnstert.2010.06.084.

4. Bulun SE, Yilmaz BD, Sison C, Miyazaki K, Bernardi L, Liu S, Kohlmeier A, Yin P, Milad M, Wei J. Endometriosis. Endocr Rev. [Internet]. 2019 Aug 1;40(4):1048-1079. Disponível em: https://doi:10.1210/er.2018-00242.

5. PASSOS EP. et al. Endometriose. Revista HCPA [Internet]. 2000 [Citado 2021 Fev 27];20(2),150-60. Disponível em: https://www.hcpa.edu.br/downloads/pesquisa/RevistaCi entifica/2000/2000 2.pdf

6. Berbel BT, Podgaec $S$, \& Abrão MS. Análise da associação entre 0 quadro clínico referido pelas pacientes portadoras de endometriose e o local de acometimento da doença. Revista De Medicina [Internet]. 2008 Set;87(3), 195-200. Disponivel em: https://www.researchgate.net/publication/275416745 A nalise da associacao entre o quadro clinico referido pelas pacientes portadoras de endometriose e o l ocal de acometimento da doenca

7. Lorençatto C, Vieira MJ, Pinto CL, Petta CA. Avaliação da frequência de depressão em pacientes com endometriose e dor pélvica. Rev Assoc Med Bras, [Internet].2002;48(3), 217-21. Disponível em: http://repositorio.unicamp.br/bitstream/REPOSIP/25848 11/S0104-42302002000300033.pdf

8. Arruda MS, Petta CA, Abrão MS, Benetti-Pinto CL. Time elapsed from onset of symptoms to diagnosis of endometriosis in a cohort study of Brazilian women. Human Reproduction [Internet] 2003 Abr;18(4), p.756$759 . \quad$ Disponível em https://doi.org/10.1093/humrep/deg136

9. Minson FP, Abrão MS, Sardá Júnior J, Kraychete DC, Podgaec S, Assis FD. Importance of quality of life assessment in patients with endometriosis. Rev Bras Ginecol Obstet. [Internet] 2012;34(1):11-5. Disponivel em https://doi.org/10.1590/S0100-72032012000100003

10. Dias Vila AC, Vandenberghe L, Almeida NS. A vivência de infertilidade e endometriose: pontos de atenção para profissionais de saúde. Psicologia, Saúde e Doenças [Internet]. 2010;11(2);219-228. Disponível em: https://www.redalyc.org/articulo.oa?id=36219023004. 
11. Oliveira LA, Brilhante AV, Lourinho LA. Relação entre ocorrência de endometriose e sofrimento psíquico. Rev. bras. promoç. Saúde,[Internet] 2018;31(4): 1-6. Disponível em DOI:10.5020/18061230.2018.8755.

12. Ramos EL, Soeiro V, Rios CT. Mulheres convivendo com endometriose: percepções sobre a doença. Ciência \& Saúde [Internet], 2018;11(3):190. Disponível em DOI:10.15448/1983-652X.2018.3.28681

13. Cunha MC, Carvalho JA, Albuquerque RM, Ludermir $A B$, Novaes $M$. Infertilidade: associação com transtornos mentais comuns e a importância do apoio social. Rev. psiquiatr. Rio Gd. Sul [Internet]. 2018;30 (3);201-210. Disponível em: https://doi.org/10.1590/S0101-81082008000400009.

14. Donatti L, Ramos DG, Andres MP, Passman LJ, Podgaec S. Pacientes com endometriose que utilizam estratégias positivas de enfrentamento apresentam menos depressão, estresse e dor pélvica. Einstein (São Paulo) [Internet]. 2007; 15(1);65-70. Disponível em: https://doi.org/10.1590/S1679-45082017A03911

15. Barbosa PZ, Rocha-Coutinho ML. Maternidade: novas possibilidades, antigas visões. Psicol. clin., [Internet]. 2007;19 (1), 163-185. Disponível em:https://www.scielo.br/j/pc/a/X3dyWtRFFFfy8wnyZM gzgYd/abstract/?lang=pt

16. Bento PA, Moreira MC. Quando os olhos não veem o que as mulheres sentem: a dor nas narrativas de mulheres com endometriose. Physis [Internet] 2018, 28 (3), 280-309. Disponível em https://www.scielo.br/j/physis/a/6xgnLCKJTsnwbHvg6d YPsTx/?lang=pt

17. HELMAN CG. Saúde, cultura e doença. Porto Alegre: Artmed Editora, 2009.

18. Ciconelli RM, Ferraz MB. Tradução para o português e validação do questionário genérico de avaliação de qualidade de vida medical outcomes study 36-item short-form health survey (SF-36). Rev. Bras. Reumatol [Internet].2006 Jun;39(3); p. 143-150. Disponível em: http://repositorio.unifesp.br/handle/11600/3754

19. Nnoaham KE, Hummelshoj L, Webster $P$, d'Hooghe $T$, de Cicco Nardone F, de Cicco Nardone C, Jenkinson C, Kennedy SH, Zondervan KT; World Endometriosis Research Foundation Global Study of Women's Health consortium. Impact of endometriosis on quality of life and work productivity: a multicenter study across ten countries. Fertil Steril [Internet]. 2011 Aug;96(2);366$373 . \quad$ Disponível doi:10.1016/j.fertnstert.2011.05.090.

20. Yela DA, Qualgiato IP, Benetti-Pinto CL. Quality of Life in Women with Deep Endometriosis: A Cross-Sectional Study. Rev. Bras. Ginecol. Obstet.[Internet] 2020; 42 (2); 90-95. Disponível em: https://www.scielo.br/j/rbgo/a/wZBxJjz5pg8x9GSNafhH $\mathrm{K} 7 \mathrm{Q} /$ ?lang=en
21. Florentino AV, Pereira AM, Martins JÁ, Lopes RG, Arruda RM. Quality of Life Assessment by the Endometriosis Health Profile (EHP-30) Questionnaire Prior to Treatment for Ovarian Endometriosis in Brazilian Women. Rev Bras Ginecol Obstet [Internet]; 2019;41;548-554. Disponível em: https://doi.org/ 10.1055/s-0039-1693057.

22. TROVÓ-MARQUI AB, SILVA MPC, IRIE GRF, Disfunção sexual em endometriose: uma revisão sistemática. Medicina (Ribeirão Preto), [Internet]. 2015;48(5);478-490. Disponível em: DOl: 10.11606/issn.2176-7262.v48i5p478-490.

23. DENNY E, MANN CH. Endometriosis-associated dyspareunia: the impact on women's lives. Journal Of Family Planning And Reproductive Health Care [Internet]. 2007 Jul 1, 33(3), 189-193. Disponível em: http://dx.doi.org/10.1783/147118907781004831.

24. EVANGELISTA A, DANTAS T, ZENDRON C, SOARES T, VAZ George, OLIVEIRA M.A.P. Sexual Function in Patients with Deep Infiltrating Endometriosis. The Journal Of Sexual Medicine [Internet]. Jan 2014; 11(1); 140-145. Disponível em http://dx.doi.org/10.1111/jsm.12349.

25. Haynes BR. Forming research questions. J Clin Epidemiol. 2006;59:881-6. 